\title{
Lesson of the month 1: Large vessel vasculitis - a diagnostic challenge and the role of 18-fluorodeoxyglucose positron emission tomography
}

\author{
Authors: Andrew Allard ${ }^{A}$ and Ramesh Mootoo ${ }^{B}$
}

\begin{abstract}
Large vessel vasculitis can pose a significant diagnostic challenge. It may be insidious in onset with the only presenting symptoms consisting of constitutional compromise. It may mimic other pathologies and the only serological abnormalities may be abnormal inflammatory markers. Conventional imaging modalities may not be diagnostic. We present a case of large vessel vasculitis that proved a significant diagnostic challenge with diagnosis established on $18 \mathrm{~F}$-fluorodeoxyglucose positron emission tomography (18F-FDG PET) computerised tomography (CT) imaging. This is one of five cases of large vessel vasculitis that were diagnosed in the rheumatology department at our trust over a 12-month period with diagnosis established with the use of 18F-FDG PET CT. We discuss the advantages of 18F-FDG PET CT over more conventional imaging modalities in diagnosing large vessel vasculitis.
\end{abstract}

KEYWORDS: 18F-fluorodeoxyglucose positron emission tomography, large vessel vasculitis

\section{Case history}

Non-specific constitutional symptoms can be the presenting features of numerous pathologies. We present a 61-year-old female who was unwell for 5 months with fatigue, anorexia, weight loss of $10 \mathrm{~kg}$ and a dry cough. Investigations in primary care found her to be anaemic with haemoglobin $(\mathrm{Hb}) 97 \mathrm{~g} / \mathrm{L}$ (normal range 115-165 g/L), MCV 69.4 fL (80-96 fL), elevated ferritin of $536 \mu \mathrm{g} / \mathrm{L}$ $(15-300 \mu \mathrm{g} / \mathrm{L})$ and low albumin of $27 \mathrm{~g} / \mathrm{L}(37-49 \mathrm{~g} / \mathrm{L})$, other liver function tests normal. Her globulins were $62 \mathrm{~g} / \mathrm{L}(24-27 \mathrm{~g} / \mathrm{L})$ with a diffuse hypergammaglobulinaemia and no paraproteinaemia, urine was negative for free light chains and her C-reactive protein (CRP) was $67 \mathrm{mg} / \mathrm{L}(<10 \mathrm{mg} / \mathrm{L})$. Abdominal ultrasound demonstrated a fatty liver and a $14 \mathrm{~mm}$ hyperechoic lesion suggestive, but not diagnostic, of a haemangioma. Because of the latter, computerised tomography (CT) of the abdomen was performed, which showed no liver parenchymal lesion but a markedly thickened aorta. Her CRP was persistently raised and infection screen was negative. She was referred to rheumatology because of the aortic thickening and

Authors: ASpR in rheumatology and general internal medicine, Royal National Hospital for Rheumatic Diseases, Bath, UK;

${ }^{B}$ consultant in rheumatology, Gloucestershire Royal Hospitals NHS Foundation Trust, Gloucester, UK elevated inflammatory markers and was seen in the outpatient department 5 months after the initial presentation to her primary care physician. Examination revealed a left supraclavicular bruit but no significant right to left blood pressure difference. Serology showed significantly raised inflammatory markers with CRP $98 \mathrm{mg} / \mathrm{L}$ and erythrocyte sedimentation rate $101 \mathrm{~mm} /$ hour (normal $<30 \mathrm{~mm}$ /hour). The anaemia worsened with an $\mathrm{Hb}$ of $84 \mathrm{~g} / \mathrm{L}$. Anti-neutrophil cytoplasmic antibody (ANCA) and antinuclear antibody were negative. A positron emission tomography (PET) CT scan demonstrated large vessel vasculitis with involvement of the thoracic and abdominal aorta as well as the subclavian, axillary, iliac, femoral and popliteal arteries bilaterally (Fig 1). She was commenced on prednisolone $1 \mathrm{mg} / \mathrm{kg}$ as well as methotrexate with good serological and clinical response. Time from presentation to diagnosis was 6 months.

\section{Discussion}

Large vessel vasculitis describes a group of conditions that cause inflammation of the aorta and its branches. It is an uncommon but well recognised cause of non-specific constitutional symptoms that present a significant diagnostic challenge. Causes include giant cell arteritis, Takayasu's arteritis and isolated aortitis. The latter may be associated with preceding infection and several associated organisms have been identified. ${ }^{1}$ Other conditions associated with inflammation of the large arteries include Behçet's disease, Cogan's syndrome, systemic lupus erythematosus, spondyloarthritis and sarcoidosis. Other types of vasculitis, such as the ANCA-positive vasculitides and polyarteritis nodosa, may occasionally involve the large vessels and present in a similar manner. ${ }^{2}$ In large vessel vasculitis the vessels involved are not easily accessible for biopsy; therefore, an underlying histological diagnosis is often not made - hence the term large vessel vasculitis. The diagnostic challenge occurs as patients may present with non-specific, constitutional symptoms and fever, sweats, malaise, weight loss, raised inflammatory markers and a normocytic or microcytic anaemia, these may be the only initial features.

Other features of the history and examination may give a clue to the underlying diagnosis but are easily missed. A history of claudication may raise suspicion. Right to left blood pressure differences and bruits heard over the affected arteries will support the diagnosis of large vessel vasculitis. There may be evidence of end organ dysfunction such as deteriorating renal or liver function. Autoimmune profile and ANCA screen are often negative. 
Fig 1. PET CT imaging of a patient with large vessel vasculitis. A - normal PET CT; $B$ - patient's PET CT showing increased uptake in the ascending aorta extending to the right axillary artery as well as in the descending aorta. $\mathrm{CT}=$ computerised tomography; $\mathrm{PET}=$ positron emission tomography
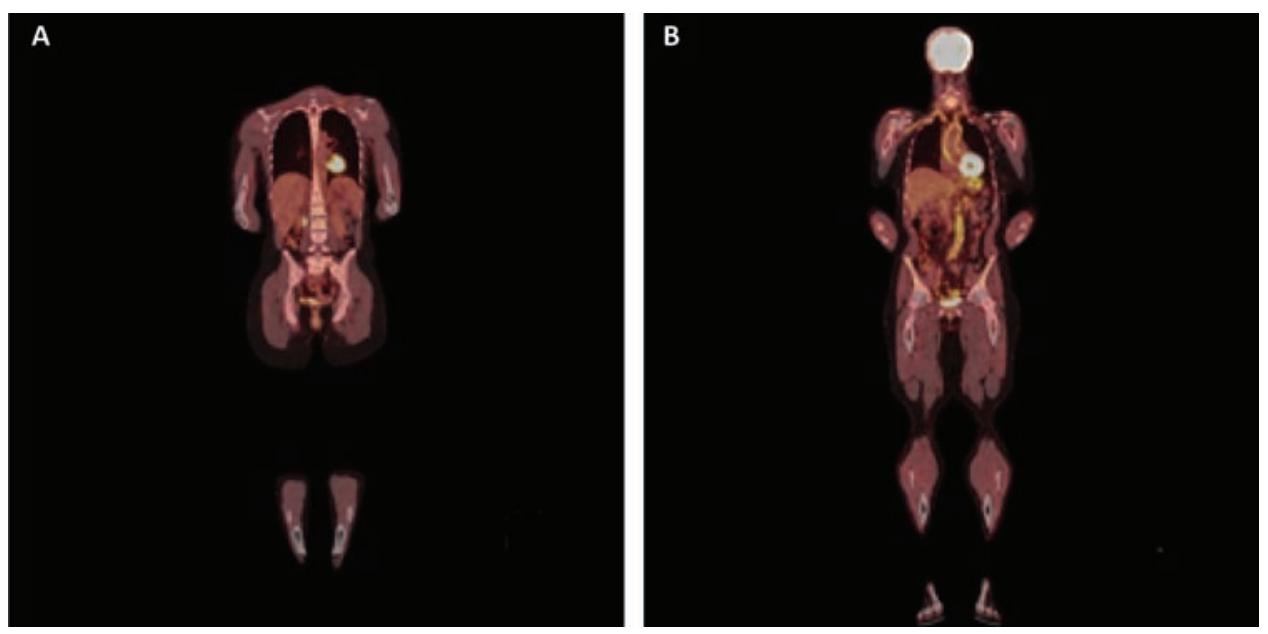

Various imaging modalities have been used to aid investigation. Ultrasonography of the temporal arteries may suggest arteritis and identify a site for biopsy to confirm giant cell arteritis as an underlying cause, but a negative scan does not exclude the diagnosis. The percentage of patients who may have positive scans when presenting without temporal headaches is unknown. Echocardiography or ultrasonography of the abdominal aorta may identify aneurysmal changes and suggest inflammation but are not diagnostic. CT angiography (CTA) can demonstrate structural changes resulting in luminal narrowing suggestive of vasculitis but it does not confirm the presence of inflammation. There may be difficulties distinguishing luminal narrowing secondary to vasculitis and narrowing due to atherosclerotic change. Magnetic resonance angiography can demonstrate structural changes with a comparable diagnostic value to CTA but may provide more detailed assessment of cardiac and aortic root pathology. ${ }^{3}$

PET CT is less widely available than these imaging modalities. It carries a 1.5-2 times greater dose of radiation than conventional CT chest abdomen pelvis depending on the protocol. ${ }^{4}$ It does, however, provide a clear, non-invasive method for confirming a diagnosis that is otherwise difficult to make with good sensitivity and specificity. ${ }^{5,6}$

Delayed diagnosis of large vessel vasculitis could result in vessel occlusion or aneurysmal formation and lead to significant mortality ranging from $3-21 \%{ }^{7}$ Treatment of large vessel vasculitis consists of high-dose corticosteroids with or without immunosuppression to induce remission and then maintenance treatment for up to 2 years before considering tapering treatment. ${ }^{8}$

This case is one of five cases of large vessel vasculitis, presenting only with constitutional symptoms and raised inflammatory markers to our rheumatology department in a 12-month period. Patients presented via a variety of pathways including to primary care and the acute medical take. All diagnoses were made with the assistance of PET CT. The time to diagnosis ranged from 6 weeks to 18 months and averaged just over 8 months.

\section{Conclusion}

This case demonstrates the importance of PET CT in establishing a diagnosis of large vessel vasculitis. Delay in diagnosis may result from lack of access to the appropriate imaging or failure to consider the diagnosis in the differential. Although relatively rare, large vessel vasculitis is an important cause of unexplained constitutional symptoms. This report aims to highlight the potential use of PET CT in the investigation of unexplained constitutional symptoms and the importance of considering large vessel vasculitis in the differential diagnosis. Delayed diagnosis of large vessel vasculitis has the potential to cause significant morbidity and mortality.

\section{Conflicts of interest}

The authors have no conflicts of interest to declare.

\section{Acknowledgement}

Written consent was obtained from the patient for publication of the clinical details and images in this article.

\section{References}

1 Lopes RJ, Almeida J, Dias PJ, Pinho P, Maciel MJ. Infectious thoracic aortitis: a literature review. Clin Cardiol 2009;32:488-90.

2 Miller DV, Maleszewski JJ. The pathology of large-vessel vasculitides. Clin Exp Rheumatol 2011;29(Suppl 64):S92-8.

3 Hartlage GR, Palios J, Barron BJ et al. Multimodality imaging of aortitis. JACC Cardiovasc Imaging 2014;7:605-19.

4 Huang B, Law MW, Khong PL. Whole-body PET/CT scanning: estimation of radiation dose and cancer risk. Radiology 2009;251:166-74.

5 Danve A, O'Dell J. The role of $18 \mathrm{~F}$ fluorodeoxyglucose positron emission tomography scanning in the diagnosis and management of systemic vasculitis. Int J Rheum Dis 2015;18:714-24.

6 Soussan M, Nicolas P, Schramm C et al. Management of large-vessel vasculitis with FDG-PET: a systematic literature review and metaanalysis. Medicine 201594:e622.

7 Li J, Zhu M, Li M et al. Cause of death in Chinese Takayasu arteritis patients. Medicine 2016;95:e4D69.

8 Mukhytyr C, Guillevin L, Cid MC et al. EULAR recommendations for the management of large vessel vasculitis. Ann Rheu Dis 2009;68:318-23.

Address for correspondence: Dr Andrew Allard, Royal National Hospital for Rheumatic Diseases, Upper Borough Walls, Bath BA1 1RL, UK.

Email: a.allard@doctors.org.uk 